\title{
Chip-based human liver-intestine and liver-skin co-cultures - a first step toward systemic repeated dose substance testing in vitro
}

Corresponding author: Ilka Maschmeyer, ilka.maschmeyer@TissUse.de, Tel: 0049-30 31427907

\section{Abstract}

Systemic repeated dose safety assessment and systemic efficacy evaluation of substances are currently carried out on laboratory animals and in humans due to the lack of predictive alternatives. Relevant international regulations, such as OECD and ICH guidelines, demand long-term testing and oral, dermal, inhalation, and systemic exposure routes for such evaluations. So-called "human-on-achip" concepts are aiming to replace respective animals and humans in substance evaluation with miniaturized functional human organisms. The major technical hurdle toward success in this field is the life-like combination of human barrier organ models, such as intestine, lung or skin, with parenchymal organ equivalents, such as liver, at the smallest biologically acceptable scale. Here, we report on a reproducible homeostatic long-term co-culture of human liver equivalents with either a reconstructed human intestinal barrier model or a human skin biopsy applying a microphysiological system. We used a multi-organ chip (MOC) platform, which provides pulsatile fluid flow within physiological ranges at low media-to-tissue ratios. The MOC supports submerse cultivation of an intact intestinal barrier model and an air-liquid interface for the skin model during their co-culture with the liver equivalents respectively at $1 / 100.000$ the scale of their human counterparts in vivo. To increase the degree of organismal emulation, microfluidic channels of the liver-skin co-culture could be successfully covered with human endothelial cells, thus mimicking human vasculature, for the first time. Finally, exposure routes emulating oral and systemic administration in humans have been qualified by applying a repeated dose administration of a model substance - troglitazone - to the chip-based co-cultures.

\section{Keywords}

Human barrier models, intestine model, skin equivalent, 3D tissue culture, organ-on-a-chip, humanon-a-chip, microphysiological system, alternatives to animal testing, vasculature, systemic drug testing

\section{Abbreviations}

$\mu$ miv marticle imaging velocimetry

BSEP bile salt export pump

CMOS complementary metal-oxide-semiconductor

CPS-1 carbamoyl-phosphate synthase 1 


$\begin{array}{ll}\text { CYP } & \text { cytochrome P450 } \\ \text { DMSO } & \text { dimethyl sulfoxide } \\ \text { ECGM-MV2 } & \text { Endothelial Cell Growth Medium MV2 } \\ \text { HDMEC } & \text { human dermal microvascular endothelial cells } \\ \text { HHSteC } & \text { human hepatic stellate cells } \\ \text { ICH } & \text { international conference on harmonization } \\ \text { LC-MS/MS } & \text { liquid chromatography tandem mass spectrometry } \\ \text { MMP7 } & \text { matrix-metalloproteasen 7 } \\ \text { MOC } & \text { multi-organ chip } \\ \text { MPS } & \text { microphysiological system } \\ \text { MRP-1 } & \text { multidrug resistance-associated protein 1 } \\ \text { MRP-2 } & \text { multidrug resistance-associated protein 2 } \\ \text { MUC2 } & \text { mucin 2 } \\ \text { NaK-ATPase } & \text { sodium-potassium pump } \\ \text { OECD } & \text { Organisation for Economic Co-operation and Development } \\ \text { PDMS } & \text { polydimethylsiloxane } \\ \text { PPARa } & \text { peroxisome proliferator-activated receptor alpha } \\ \text { PPARg } & \text { peroxisome proliferator-activated receptor gamma } \\ \text { qRT-PCR } & \text { real-time qPCR } \\ \text { RBC } & \text { human red blood cells } \\ \text { SI } & \text { small intestine } \\ \text { SLC5A1 } & \text { sodium/glucose cotransporter 1 } \\ \text { TBP } & \text { TATA-binding protein } \\ \text { TEER } & \text { transepithelial electrical resistance } \\ \text { TUNEL } & \text { TdT-mediated dUTP-digoxigenin nick end labeling } \\ \text { VWF } & \text { Von Willebrand factor } \\ \text { ZO-1 } & \text { zonula occludens 1 } \\ & \end{array}$

\section{Introduction}

Strategies to develop "human-on-a-chip" platforms are applying microphysiological systems (MPS) to the in vitro combination of miniaturized human organ equivalents into miniature functional human organisms[1-4]. It has been widely accepted that systems with at least ten organs are needed to assemble human organismal homeostasis at a level of complexity comparable to that of human or animal bodies. These aim to replace systemic toxicity testing and efficacy assessment of therapeutic agents, food additives, chemicals, or environmental pollutants in laboratory animals, and to predict human safety and efficacy data prior to substance exposure to humans[5]. Numerous microphysiological "organ-on-a-chip" devices have been developed and applied to the culture of single organ tissues under dynamic conditions at miniature scale, thus overcoming the artificial conditions of static tissue cultures. Their main advantage lies in providing proper dynamic mechanical and chemical cues to the tissues, thus emulating the real situation of the tissue microenvironment in vivo. They have been reviewed extensively in the past $[1,2,6]$. However, up to the present day, only a 
few multi-organ chip (MOC) concepts have been translated into MPSs, combining several tissue types[7-11]. In addition to the physiological way of mechanically and chemically coupling each individual tissue, these devices use their microfluidic channel systems to physically combine different chip-based cell culture compartments with each other as a first engineering step toward organisms on a chip. The major technical hurdle toward success in this field is the life-like combination of human barrier organ models with parenchymal organ equivalents at the smallest biologically acceptable scale. Furthermore, human cell lines in suspension or monolayers, rather than primary cells and tissues with 3D architectures, are used to mimic organ tissues in such systems. None of these existing multi-organ systems address the challenges of air-liquid interfaces for barrier models, such as skin or lung. Finally, all of these microphysiological multi-organ systems lack the human endothelial cell coverage of the microfluidic channels. Organs in the human body are interconnected by a vascular network entirely lined by human endothelial cells, representing nature's blood-tissue barrier. The endothelial cell layer communicates with the tissue and signals into the blood stream to recruit, for example, leucocytes into a region of local damage in the organism. Finally, a closed endothelial cell layer prevents blood cells from bleeding into tissue and clotting. Different approaches to establish human vasculature in vitro at a mini-scale through the so-called BioVaSc technology[12-14] and at a microscale on chips[15-18] have been published in the past. Such endothelialization would be preferable to emulate the biological impact of human vasculature on systemic organismal homeostasis in microphysiological MOC systems. In order to overcome the aforementioned challenges, we developed a novel MOC platform allowing the culture of reconstructed parenchymal and barrier organ equivalents or the respective biopsy tissues over long periods at a scale 100.000 -fold smaller than the original organs. As a proof of concept, we were able to demonstrate a stable four-week homeostasis between human 3D liver spheroids, composed of HepaRG cells and human primary stellate cells, and skin prepuce biopsies in the microphysiological MOC system[19]. Moreover, we developed a method to fully cover the microfluidic channel system of our MOCs with human microvascular endothelial cells under physiological pulsatile flow conditions[18].

We here report, for the first time, the application of the MOC system for the co-culture of human 3D liver spheroids with a second barrier model - human intestine - composed of primary human intestinal epithelial cells. Furthermore, we improved the established co-culture of liver spheroids and skin biopsies by endothelialization of the entire microchannel system of the chip, mimicking human vasculature. Finally, we emulate systemic, oral or dermal routes of repeated dose substance exposure in the two co-cultures over an exposure time of nine to eleven days. 


\section{Materials and Methods}

Design and fabrication of the microphysiological system

Multi-organ chips were produced, as described by Wagner et al.[19]. In brief, a single polydimethylsiloxane (PDMS) layer, height $2 \mathrm{~mm}$, containing the respective arrangement of channels, micropumps and openings for culture compartments was permanently bonded to a glass microscope slide with a footprint of $75 \times 25 \mathrm{~mm}$ (Menzel, Braunschweig, Germany) by low pressure plasma oxidation (Femto; Diener, Ebhausen,Germany), thus forming the respective fluid-tight microfluidic MOC device with standard channel heights of $100 \mu \mathrm{m}$. The peristaltic on-chip micropump was modified after Wu and co-workers [20], and represents three $500 \mu \mathrm{m}$ thick PDMS membrane valves in a row. They are consecutively actuated by applying pressure. The microfluidic layout of the MOC used in this study consisted of two culture compartments per co-culture circuit (Fig. 1C). Each culture compartment holds a maximum of $300 \mu \mathrm{l}$ medium. The direct exposure of tissue to fluid flow in the liver spheroid culture compartment (left side) and the alternative co-culture setting in a Transwell ${ }^{\circledR}$ separating tissues from the fluid flow in the barrier organ culture compartment (right side) by a porous polycarbonate membrane (thickness $10 \mu \mathrm{m}$, pore size $0.4 \mu \mathrm{m}$ ) are highlighted.

\section{Management of microfluidics}

The MOC platform provides a number of tools, e.g. pumping frequency and peristaltic valve pressure, to adjust fluid flow and, consequently, mechanical forces according to the physiology of the respective organ equivalents. Micro particle imaging velocimetry ( $\mu$ PIV) has been routinely established to set process parameters for physiological MOC operation[18]. We improved the $\mu$ PIV technology here so that it became biocompatible for measurements in sterile, endothelial cell covered MOC circuits. Process parameters supporting a median shear stress below $2 \mathrm{dyn} \mathrm{cm}^{-2}$ in position A of each microfluidic circuit (Fig. 1C) had to be selected[21]. This would shield the liver equivalents in the subsequent culture compartment from high mechanical stresses. Human red blood cells (RBCs) instead of synthetic polymeric particles were established for that type of imaging velocimetry. In brief, human RBCs were obtained from donors by venepuncture. Plasma was discarded and the cells were suspended in $0.9 \%$ sodium chloride solution. A hematocrit of $2.5 \%$ was chosen for the RBC imaging solution. An amount of $300 \mu \mathrm{l}$ of the solution was gently filled into each insert area of the circuit of a fully endothelialized MOC. Subsequently, the MOC was operated at different process regimens. The circulating RBCs were recorded in a region of interest around position A by a high-speed CMOS-camera ( $\mathrm{HXC40}$, Baumer) attached to a standard inverted microscope (2.5 x magnification). At point $A$ the cross-section of the channel was $100 \mu \mathrm{m}$ high and $500 \mu \mathrm{m}$ wide. The y-resolution was reduced to $112 \mathrm{px}$ framing only the channel boundaries to raise the frame rate above $2,900 \mathrm{fps}$. The pixel size was determined to be $4.32 \mu \mathrm{m} \mathrm{px}^{-1}$. The RBC 
displacement was analyzed in consecutive images by PIVlab[22,23] an open-source tool for MATLAB. In order to derive only the maximum velocity in the $x$-direction of the central part of the images, one quarter was omitted on either side in the y-direction. Profiles of the pulsatile flow in position A were recorded (Fig. 1D). From this a displacement curve was derived. Calculating a linear correlation gave rise to the median flow rate and the median shear stress at the channel walls in position $A$, eventually. Essential mathematics have been described by us earlier [18]. Process parameters ensuring a median shear stress of less than $0.7 \mathrm{dyn} \mathrm{cm}^{-2}$ were identified and set for the respective coculture experiments.

Cell and tissue sources

The human HepaRG cell line was obtained from Biopredic International (Rennes, France). Human primary hepatic stellate cells (HHSteC) were purchased from ScienCell Research Laboratories (Carlsbad, CA, USA). Reconstructed human small intestinal barrier models in cell culture inserts of the 96-well size were constructed by MatTek Corporation (Ashland, MA, USA). Human juvenile prepuce from 6 different donors of the age of 3 to 5 years was obtained from a pediatric surgery after routine circumcisions, with informed consent and ethics approval (Ethic Committee Charité University Medicine, Berlin, Germany), in compliance with the relevant laws. Skin biopsies and human dermal microvascular endothelial cells (HDMEC) were derived from such prepuce samples.

Reconstruction of the intestine barrier model

Intestine barrier models, produced at MatTek Corporation, were established using primary small intestine (SI) epithelial cells from the ileum section of small intestine tissue obtained from a 19 year old Caucasian female donor with no history of gastrointestinal disease. The tissues were obtained with informed consent. The cells were screened for HIV, HBV, HCV and mycoplasma prior to use and were found to be negative. Small intestinal epithelial cells were harvested using enzymatic tissue dissociation and primary cell culture techniques. The cells were expanded in culture and seeded directly onto 96-well cell culture inserts, raised to the air-liquid interface, and cultured using a specially formulated culture medium (MatTek Corporation) designed to induce differentiation over a period of 14 days. The necessary amount of insert-based intestinal barrier cultures were released for a two-day overseas shipment at $4^{\circ} \mathrm{C}$ after transepithelial electrical resistance (TEER) levels reached a stable range of $50-180 \Omega * \mathrm{~cm}^{2}$ mimicking the physiologic human SI microenvironment[24-26]. Upon reception, the cell culture inserts were transferred into the respective number of MOC systems. Insert-holders ensured a precise positioning of the barrier models at a height of $100 \mu \mathrm{m}$ above the bottom glass slide of each MOC-circuit to ensure free media passage below the intestinal barrier models. 
Preparation of the skin biopsy barrier

Prepuce samples with an average size of $2.5 \mathrm{~cm}^{2}$ were stored and transported in $10 \mathrm{ml}$ phosphatebuffered saline (PBS) at $4{ }^{\circ} \mathrm{C}$ and prepared for further culture within $4 \mathrm{~h}$ following surgery. They were subsequently incubated in $80 \%$ ethanol for $30 \mathrm{~s}$. Samples with an average thickness of $2 \mathrm{~mm}$ were punched to provide biopsies of $5 \mathrm{~mm}$ diameter. Samples were loaded with the epidermal-side up into 96-well Transwell ${ }^{\circledR}$ (Corning, Lowell, MA, USA) inserts and were then integrated into the respective MOC culture compartments.

De novo formation of liver equivalents

Cell culture components were purchased from PAA Laboratories (GE Healthcare Europe GmbH, Vienna, Austria) and cultures were incubated at $37^{\circ} \mathrm{C}$ and $5 \% \mathrm{CO}_{2}$, unless otherwise stated.

HepaRG cells were maintained, as described by Gripon et al.[27]. Briefly, cells were cultured in HepaRG medium, consisting of William's Medium E supplemented with $10 \%$ fetal calf serum, 100 units per ml penicillin, $100 \mu \mathrm{g} \mathrm{ml}^{-1}$ streptomycin, $5 \mathrm{\mu g} \mathrm{ml}^{-1}$ human insulin, $2 \mathrm{mM} \mathrm{L-glutamine,} \mathrm{and} 5 \mathrm{x}$ $10^{-5} \mathrm{M}$ hydrocortisone hemisuccinate (Sigma-Aldrich, St. Louis, MO, USA). Medium was renewed every two or three days. Induction of differentiation was initiated by maintaining the cells in growth medium for two weeks in order to reach cellular confluence, according to the manufacturer's instructions. Differentiation medium containing 2\% dimethyl sulfoxide (DMSO; Carl Roth GmbH, Karlsruhe, Germany) was then added for a cultivation period of two weeks. When differentiation was completed, the cells were used for further experiments. Human stellate cells were expanded in Stellate Cell Media, provided by ScienCell Research Laboratories (Carlsbad, CA, USA), on poly-Llysine-coated T75 tissue culture flasks (ScienCell). Cells were harvested for further use at $80 \%$ confluence.

Human liver spheroids were formed combining HepaRG cells and human stellate cells, as described previously[19], using Perfecta3D ${ }^{\circledR}$ 384-Well Hanging Drop Plates (3D Biomatrix, Ann Arbor, MI, USA). Briefly, $20 \mu \mathrm{l} \mathrm{HepaRG} \mathrm{medium} \mathrm{containing} 4.8 \times 10^{4}$ hepatocytes and $0.2 \times 10^{4} \mathrm{HHSteC}$ were pipetted to each access hole. After two days of hanging drop culture, spheroids were transferred to ultra-low attachment 24-well plates (Corning). Twenty spheroids were collected together to form a single liver equivalent in the respective culture compartment of an MOC insert, as described in Wagner et al., 2013[19].

Isolation of endothelial cells

Human dermal microvascular endothelial cells were isolated from prepuce samples as described previously by Schimek et al.[18]. Briefly, samples were cleaned in $80 \%$ ethanol, subcutaneous tissue 
was removed and samples were incubated in $5 \mathrm{mg} \mathrm{ml}-1$ dispase II solution (Sigma-Aldrich, Schnelldorf, Germany) at $4{ }^{\circ} \mathrm{C}$ for 15 to $18 \mathrm{~h}$. The dermis was subsequently incubated in $4 \mathrm{mg} \mathrm{ml}-1$ Collagenase NB 4 solution (Serva, Heidelberg, Germany) at $37{ }^{\circ} \mathrm{C}$ for $75 \mathrm{~min}$. The cell solution was filtered through a $70 \mu \mathrm{m}$ nylon filter (Corning, Lowell, MA, USA) and centrifuged at $300 \mathrm{~g}$ for $5 \mathrm{~min}$. The resulting cell pellet was resuspended in Endothelial Cell Growth Medium MV2 (ECGM-MV2; PromoCell, Heidelberg, Germany) supplemented with Supplement-Pack MV2 (PromoCell, Heidelberg, Germany), $1 \%$ penicillin-streptomycin and $0.05 \%$ fungizone. The cells were seeded into a T-75 flask and grown in $5 \% \mathrm{CO} 2$ at $37{ }^{\circ} \mathrm{C}$. Cells were purified by magnetic associated cell sorting (MACS) twice for endothelial specific surface marker PECAM-1 (Miltenyi Biotec, Bergisch Gladbach, Germany), according to the manufacturer's instructions.

Endothelialization of multi-organ chips

Prior to seeding the endothelial cells into the MOC, each circuit was flushed with ECGM-MV2 and incubated statically for three days in $5 \% \mathrm{CO}_{2}$ at $37^{\circ} \mathrm{C}$, as described by Schimek et al[18]. The HDMECs were harvested from expansion cultures and cell suspension was concentrated by centrifugation. Cell viability was $>90 \%$ for all experiments. Centrifuged cells were resuspended with complete ECGM$\mathrm{MV} 2$ to a final concentration of $2 \times 10^{7}$ cells $\mathrm{ml}^{-1}$. Afterwards, the cell suspension was injected through one of the two compartments of each circuit into the system using a $1 \mathrm{ml}$ syringe. After even cell infusion into both circuits the device was incubated in $5 \% \mathrm{CO}_{2}$ at $37{ }^{\circ} \mathrm{C}$ under static conditions for $3 \mathrm{~h}$ to allow the cells to attach to the channel walls. An amount of $300 \mu \mathrm{l}$ fresh medium was added to each compartment and then flushed through the PDMS channels using the on-chip micropump of each circuit. A frequency of $2 \mathrm{~Hz}$ was applied to the microvascular circuits of the MOCs for continuous dynamic operation. The frequency was defined as the amount of pulses of the pump cycle per second.

\section{Chip-based co-cultures}

Ten MOC circuits were loaded with an intestine barrier insert and liver equivalent and eight endothelialized MOC circuits were loaded with a skin biopsy insert and a liver equivalent for subsequent co-culture. The height of the tissue and the amount of medium added to the cell culture inserts enabled the tissues to be at the air-liquid interface (skin) or submerged in media (intestine). Co-cultures were cultured in $450 \mu \mathrm{l}$ circulating medium, an additional $50 \mu \mathrm{l}$ of medium was added on top of intestinal tissues, leading to a total volume of $500 \mu \mathrm{l}$ in intestinal and liver tissue cultures and $450 \mu \mathrm{l}$ in skin and liver co-cultures. $250 \mu \mathrm{l}$ of this medium was replaced daily and supernatants were collected to determine the lactate release and the glucose consumption. At the end of the fourteen- 
day co-culture, cell viability and protein expression were analyzed by immunohistochemistry and qRT-PCR techniques.

\section{Model substance}

We used troglitazone, a substance inducing metabolic activities and, finally, toxicity in HepaRG cells, to challenge the co-cultures at repeated dose regimens. Troglitazone (Sigma-Aldrich Chemie GmbH, Munich, Germany) was dissolved in DMSO, stored frozen at a concentration of $20 \mathrm{mM}$ until use, and then diluted in culture medium to a level of $0.05 \%$ DMSO. Medium containing $0.05 \%$ DMSO was used for control cultures.

Oral exposure to liver-intestine co-cultures

Oral substance exposure to the MOC co-cultures was modeled by administration of $50 \mu \mathrm{l}$ of a $100 \mu \mathrm{M}$ troglitazone solution into the apical compartment of the tissue culture inserts on top of the human intestinal barrier. A complete penetration of troglitazone through the intestine, therefore, would result in a daily application of a systemic concentration of $10 \mu \mathrm{M}$. The daily administration started after a three-day administration-free adaptation period and was performed over a period of 11 days. Experiments were finished at day 14 and tissues were removed from the MOC. Cell viability and protein expression were assessed by immunohistochemistry and qRT-PCR. All experiments were performed in quadruplicate.

Systemic exposure to liver-skin co-cultures

Systemic substance exposure to the MOC was modeled by diluting $2.5 \mu \mathrm{l}$ of a $20 \mathrm{mM}$ troglitazone solution in cell culture medium and administering it to each MOC circuit, resulting in a final systemic concentration of $50 \mu \mathrm{M}$ troglitazone. The daily treatment started after a five-day administration-free adaptation period and was performed over a period of nine days. Experiments were finished at day 14 and tissues were removed from the MOC. Cell viability and protein expression were assessed by immunohistochemistry and qRT-PCR. All experiments were performed in quadruplicate.

\section{Co-culture analyses}

Tissue metabolic activity was monitored daily by the measurement of glucose and lactate in the media supernatants. Therefore, the Glucose LiquiColor ${ }^{\circledR}$ Oxidase kit (Stanbio Laboratory, Boerne, USA) was used with minor modifications: An amount of $100 \mu \mathrm{l}$ of reagent was used and $1 \mu \mathrm{l}$ of sample was added. Lactate concentration of the medium was measured using the LAC 142 kit (Diaglobal, Berlin, Germany), according to the manufacturer's instructions, with minor modifications 
based on our own standard curves: An amount of $99 \mu \mathrm{l}$ of the reagent was mixed with $1 \mu \mathrm{l}$ of sample and absorbance was measured at $520 \mathrm{~nm}$, using medium as a reference.

Immunohistochemical end-point analyses were performed by staining the tissue sections for cytokeratin $8 / 18$, Von Willebrand factor (VWF), vimentin, cytokeratin 10 , cytokeratin 15 , sodiumpotassium pump (NaK-ATPase), and multidrug resistance-associated protein 2 (MRP-2). Briefly, representative central cryosections of the tissues were fixed in acetone at $-20{ }^{\circ} \mathrm{C}$ for $10 \mathrm{~min}$ and blocked with $10 \%$ goat serum in PBS. Liver sections were then incubated with mouse anti-human cytokeratin 8/18, rabbit anti-human vimentin (both Santa Cruz Biotechnology, Heidelberg, Germany) and rabbit anti-human vWF antibody (Abcam, Cambridge, UK) for $1.5 \mathrm{~h}$ and washed with PBS. Goat anti-mouse IgG Alexa Fluor ${ }^{\circledR} 594$ and goat anti-rabbit IgG Alexa Fluor ${ }^{\circledR} 488$ (all purchased from Life Technologies, Darmstadt, Germany) were used for visualization. Skin tissues were stained for mouse anti-human cytokeratin 10 (Merck-Millipore, Darmstadt, Germany) and rabbit anti-human cytokeratin 15 (Abcam). Small intestinal epithelial tissue sections were stained for rabbit anti-human NaK-ATPase (Abcam), mouse anti-human Cytokeratin 8/18 and rabbit anti-human MRP-2 (both Santa Cruz Biotechnology). Staining procedures were the same as for the liver tissue. Goat anti-rabbit IgG Alexa Fluor ${ }^{\circledR} 594$ and goat anti-rabbit FITC (both purchased from Life Technologies) were used for visualization of skin and intestinal tissues.

Furthermore, apoptosis and proliferation were analyzed by immunohistological end-point stainings at the end of each MOC experiment using TUNEL (TdT-mediated dUTP-digoxigenin nick end labeling)/Ki67 markers. Briefly, representative eight-micron cryostat central sections of the tissue were stained for apoptosis using the TUNEL technique (ApopTag1 Peroxidase In Situ Apoptosis Detection Kit, Merck Millipore, Darmstadt, Germany), according to the manufacturer's instructions. The apoptosis staining was combined with a nuclear stain, Hoechst 33342 (Life Technologies), the antibody Ki67 (rabbit anti-human, Abcam) and its respective secondary antibody (goat anti-rabbit IgG Alexa Fluor ${ }^{\circledR}$ 594, Life Technologies).

Real-time qPCR endpoint analyses were performed to evaluate gene transcription at mRNA level after the MOC cultures were stopped. Therefore, tissue equivalents were collected for RNA isolation using the RNeasy Mini Kit (Qiagen, Hilden, Germany). cDNA was synthesized by reverse transcription of $400 \mathrm{ng}$ total RNA (TaqMan1, Roche Diagnostics, Mannheim, Germany). Real-time qPCR experiments were conducted using the Stratagene system (Agilent Technologies, Böblingen, Germany) and the SensiFast SYBR No-ROX One-Step Kit (Bioline, Luckenwalde, Germany), according to the manufacturer's instructions. The real-time qPCR primers are shown in Table 1. 
The barrier function of the small intestinal epithelial tissue was monitored by TEER using the Millicell ERS-2 Volt-Ohm Meter (Millipore Co., Bedford, USA) and were monitored at the beginning of the experiment and at the last day of experiment. Millicell inserts were removed from the MOC for the measurement and TEER analysis was performed, as described by the manufacturer. TEER was expressed as $\Omega^{*} \mathrm{~cm}^{2}$ based on the area of transwell plate inserts after subtracting the resistance of the supporting filter from the reading and multiplying it by the surface area of the monolayer.

Liquid chromatography tandem mass spectrometry analysis of troglitazone LC-MS/MS analysis of troglitazone from media supernatants was performed using a modular HPLC system (binary pump: LC-20AD, Shimadzu, Duisburg, Germany) coupled to a QTRAP ${ }^{\circledR} 5500$ mass spectrometer (AB Sciex, Darmstadt, Germany). Chromatographic separation was achieved using an OTU LipoMare $C_{18}$ column $(150 \times 4.6 \mathrm{~mm} ; 5 \mu \mathrm{m}$; Application \& Chromatography, Oranienburg, Germany). The injection volume was $10 \mu \mathrm{L}$ and the mobile phase flow rate was $500 \mu \mathrm{L} \mathrm{min}^{-1}$. Mobile phase A consisted of water and mobile phase B of methanol. Gradient elution was achieved by ramping from $40 \%$ B to $90 \%$ B within $10 \mathrm{~min}$, followed by a holding step at $90 \%$ B for $3 \mathrm{~min}$ and subsequent re-equilibration. All samples were diluted 1:500 and syringe filtrated $(0.2 \mu \mathrm{m}$, nylon) prior to injection. For MS/MS detection negative electrospray ionization multiple-reaction monitoring mode was used. Curtain gas was maintained at 35 psi. Optimized ion spray voltage was $4000 \mathrm{~V}$ and the source temperature was $500^{\circ} \mathrm{C}$. Quantifier ion transition was $\mathrm{m} / \mathrm{z} 440 \rightarrow 397$, qualifier ion transitions were $\mathrm{m} / \mathrm{z} 440 \rightarrow 145$ and $\mathrm{m} / \mathrm{z} 440 \rightarrow 117$.

\section{Statistical analysis}

Statistical analysis of the data was performed by t-test following the Welch test to determine significant differences among means using GraphPad software (Prism 6). Differences were considered significant at $p<0.05$. Data are expressed as the mean of four independent experiments performed in quadruplicate.

\section{Results and Discussion}

The MOC platform presented here enables the integration of biological barriers into dynamic multitissue system. The microfluidic design consists of an on-chip micropump reproducibly operating a circulation system interconnecting two organ culture compartments, each the size of a single well of 
a standard 96-well microplate. The integration of industrially available cell culture inserts allows the cultivation of tissues at an air-liquid or liquid-liquid interface. Well-established tissue models of, for example, human skin, intestine and lung can be integrated into the MOC, enabling a mechanical coupling and molecular crosstalk of the tissues with secondary organs. The secondary organ, e.g. a liver equivalent, can be placed into the second culture compartment. The widening of the channels in the culture compartments reduces the shear stress and the fluid flow in the culture area, avoiding the flushing of the tissues. To assure that the tissues do not get flushed away during peristaltic pumping, daily microscopic inspection was performed. Figure $1 \mathrm{~A}$ depicts an exemplary experimental set-up of a liver-intestine co-culture and figure 1B the experimental set-up of a skin-liver co-culture. Substance application for drug testing can be performed either systemically, by applying the substance directly to the media circuit, or apically, on the intestine. It could be demonstrated by $\mu$ PIV using RBCs, that in position A (Fig. 1C) of an endothelialised MOC circuit, the median shear stress did not exceed $0.7 \mathrm{dyn} \mathrm{cm}^{-2}$ at pump frequencies of $2 \mathrm{~Hz}$, resulting in a median flow rate of $2.6 \mu \mathrm{l} \mathrm{min}{ }^{-1}$. Therefore, the on-chip micropump was set at a $2 \mathrm{~Hz}$ frequency over the whole period of the respective experiments.

\section{Fourteen-day performance of liver-intestinal co-cultures in the MOC}

Current intestinal in vitro tests are based on monolayer cultures of immortalized cell lines, mainly Caco-2, HT-29 or T84 cell lines obtained from human colorectal adenocarcinomas. However, immortalized cell lines do not recapitulate the 3D small intestine tissue architecture and show a 100fold difference in pharmaceutical permeability coefficients compared to in vivo intestinal epithelium[28]. Therefore, the drug metabolism and absorption of these intestine models do not represent the in vivo situation correctly. In addition to using primary cells, it was shown recently that a dynamic microenvironment is critical to elicit in-vivo-like physiological responses[29]. Therefore, in this study, a human primary small intestinal equivalent was combined with a liver equivalent in the microfluidic circuit of our MOC. The liver and primary intestine equivalents were kept viable in this co-culture for 14 days. The cultures were sustained by $500 \mu \mathrm{l}$ medium which was partially replaced every $24 \mathrm{~h}$.

The small intestine model showed columnar epithelial cell morphology similar to in vivo human small intestine and had a height of up to $140 \mu \mathrm{m}$. Exposure to a higher pressure and an additional flow on the apical side of the intestinal tissue increased the height of columns to $250 \mu \mathrm{m}$ in an intestinalmono-culture (data not shown). In vivo, proliferating cells are located in the crypt base and give rise to proliferating progenitor cells which migrate upwards. Consistently, most proliferating cells 
(positive for Ki67) in the cultured intestinal tissue were located at the lowest layer, with only few proliferating cells at upper levels (Fig. 2A). Conventional CaCo-2 monolayers cultured in transwells have been reported to have unphysiologic tight junctions and therefore too high TEER values (TEER $\sim 300-600 \Omega * \mathrm{~cm}^{2}$ ) compared to physiologic TEER values ranging from $50-120 \Omega * \mathrm{~cm}^{2}$ in human small and large intestine[24,25]. In comparison, our primary intestinal epithelial tissues could keep a constant close to physiologic TEER values during the 14-day culture in the MOC (starting with TEER values of $50-180 \Omega * \mathrm{~cm}^{2}$ and ending with TEER values of $70-180 \Omega^{*} \mathrm{~cm}^{2}$ ). In Caco-2 cells, the expression of the $A B C$ membrane transporter superfamily in immuno-histology staining could not be observed under static conditions[13]. In comparison, we could detect the expression of MRP-2 in our intestinal tissues (Figure 2B). However, a polarisation of the intestinal tissue could not be achieved and MRP-2 is expressed in every cell of the tissue. The influence of a different medium on the apical and the basolateral side of the tissue and a second perfusion on the luminal side of the tissue, might help to initiate a polarisation as seen by Schanz et al.[13] on the example of MDR-1. Immunofluorescence staining revealed the expression of active transporter pumps, such as the sodium-potassium pump (Fig. 2C). Cytokeratin 8/18 expression proves their epithelial origin. The liver tissues still showed a homogenous distribution of HHSteC within the aggregates after 14 days of dynamic co-culture. No alterations in cell ratios or morphology could be observed.

Fourteen-day performance of liver-skin co-cultures in endothelialized MOCs

Liver equivalents and skin punch biopsies were co-cultivated in endothelialized MOCs under dynamic conditions for up to 14 days. Primary human endothelial cells isolated from two patients were seeded at a density of $2 \times 10^{7}$ cells $\mathrm{ml}^{-1}$ into the channels of the MOC seven days prior to adding the liver and skin tissue models to allow the establishment of a fully endothelialized microfluidic circuit. The endothelial cells covered all surfaces of the channel circuit (Fig. 3A) and produced a confluent monolayer (Fig. 3B), even after 14 days of dynamic co-culture, representing 21 days of culture in the MOC. In total, $10^{5}$ endothelial cells, $10^{6}$ liver cells and a skin biopsy of $5 \mathrm{~mm}$, having a volume of about $24 \mu \mathrm{l}$ and consisting of $5 \times 10^{6}$ skin cells, were co-cultivated in one microfluidic circuit of the MOC. These cell numbers are within the physiological range and represent roughly $1 / 100.000$ of the size of the respective counterpart organ in the human body. The cultures could be sustained at steady state conditions by a total medium volume of $500 \mu \mathrm{l}$ per MOC circuit and a daily medium exchange rate of $50 \%$. 
An interaction of the endothelial cells with the liver equivalents was observed by immunofluorescence staining showing an integration of the cells between liver spheroids in areas where they were in direct cell-cell contact (Fig 3C). However, these interactions were limited to the contact surface of the spheroids with the underlying monolayer of endothelial cells and did not show any measurable effects on the expression of liver-typical markers (data not shown). Skin punch biopsies showed a retained expression of cytokeratin 15 in basal keratinocytes and cytokeratin 10 in keratinizing and non-keratinizing stratified epithelia respectively after 14 days of culture (Fig. 3D). The continuous layer of cells observed in the stratum basale and the overlying differentiated keratinocytes of the epidermis hint at the preservation of an elementary barrier function of this model. However, the punch biopsy and the supporting cell culture insert do not form a tight connection with each other, allowing for a penetration of substances which would be applied topically into the underlying media circuit. An improvement of the model is envisaged by gluing the biopsy into the insert using fibrin or other extracellular matrix components. The integration of tissue models that were generated in porous inserts and can be purchased from various suppliers holds another possibility for a defined and tight skin barrier model to analyse effects of topical applied substances in co-culture with a second organ. Overall metabolic activity of the three-tissue cocultures, measured as glucose consumption and lactate production, were stable with only minor fluctuations from day six until the end of culture, indicating a stable artificial coexistence between the tissues (Fig. 3E).

Repeated dose substance exposure to MOC co-cultures

Troglitazone was chosen as a model substance to optimize and qualify MOC-based administration regimens equivalent to oral and systemic exposure in humans. It belongs to the thiazolidinedione class of compounds representing a family of drugs used for the treatment of noninsulin-dependent type 2 diabetes mellitus. The first commercialized thiazolidinedione drug, troglitazone (trade name: Rezulin), was withdrawn from the U.S. market after numerous reported cases of severe idiosyncratic liver damage and failures leading to liver transplantation or death. Troglitazone is rapidly absorbed with an absolute bioavailability of between 40 and 50\%[30]. During clinical trials, troglitazone was administered orally at a daily concentration of up to $600 \mathrm{mg}$ per patient. In previous studies, troglitazone was shown to also affect the metabolism of HepaRG cells in vitro[31,32]. This sensitivity provides an additional measurement of substance distribution to the liver equivalents in our cocultures. In order to reach a reproducible homeostasis in the MOC system, a three-day administration-free adaptation period is mandatory for the liver-intestine co-culture, whilst the liver- 
skin co-culture requires a five-day adaptation phase prior to administration of troglitazone. The selection of various troglitazone doses in the co-culture experiments were guided by following general assumptions: A 100.000-fold down scale of the daily patient doses corresponds to $6 \mu \mathrm{g}$ (10 $\mu \mathrm{M})$ for oral administration in the MOC. Taking into account the bioavailability, an average concentration of $5 \mu \mathrm{M}$ is expected to be a physiological range in the systemic circulation of the MOC.

Modeling oral exposure to the liver-intestine co-culture

A reproducible and easy to perform oral substance administration protocol was performed over a stretch of 11 days after a three-day administration-free adaptation period, resulting in a continuous two-week MOC performance at repeated dose testing conditions. Oral substance exposure to the MOC co-cultures was modeled by administration of the respective troglitazone solution into the apical compartment of the tissue culture inserts on top of the human intestinal barrier at a high concentration to ensure detectable effects on the liver equivalents. The substance needs to penetrate the barrier of the intestinal tissue and to be pumped to the second compartment of the $M O C$ in order to reach the liver equivalent. TEER levels of the intestinal tissues treated ranged from 70-180 $\Omega$, while control values ranged from 98-194 $\Omega$. Therefore, no significant decrease in barrier function of the intestinal barrier was observed compared to the control. The treatment of the troglitazone showed a decrease in albumin mRNA expression in liver. Similarly, the mRNA expression of carbamoyl-phosphate synthase 1 (CPS-1), a mitochondrial enzyme which transforms ammonia and bicarbonate to carbamoyl phosphate, a first step in the urea cycle to remove excess urea from cells, decreased. The mRNA expression of CYP $3 A 4$ increased (Fig. 4C), indicating an increased metabolic biotransformation from troglitazone to quinone and epoxide metabolites. These data correlate with data observed with liver single cultures[33,34] and prove that troglitazone passed the intestinal barrier, was pumped to the liver tissue and was then metabolised. In previous studies UGT 1A1 has been shown to exhibit low troglitazone glucuronosyltransferase activities[35]. In human liver microsomes, troglitazone did not inhibit bilirubin conjugation at a concentration of $100 \mu \mathrm{M}$ or less[36]. This correlates to the data of our study, showing a slight, but non-significant increase of UGT1A1 mRNA expression after the chronic application of $10 \mu \mathrm{M}$ troglitazone. The bile salt export pump (BSEP) mRNA expression did not change significantly but showed a high variance in our experiments. BSEP is involved in maintaining bile acid homeostasis in the liver. In isolated canalicular rat liver plasma membrane preparations, troglitazone competitively inhibited the ATP-dependent taurocholate transport, mediated by BSEP[37]. In contrast, it has been shown that troglitazone increases BSEP mRNA expression significantly in Huh7 liver cells after single dose application[38], whilst metabolites of troglitazone are known to reduce the mRNA expression of BSEP in in vitro cultured hepatocytes[39]. This might explain the high variance of expression after 7 day chronic 
application, where troglitazone as well as its metabolites might have influenced the expression of BSEP mRNA expression. MRP-1 and ZO-1 expression did not change after treatment with troglitazone (Fig. 4C). To access liver toxicity it would be helpful to analyse ALT and AST as liver specific lysis markers in the medium. However, the sensitivity of existing tests needs to be optimized in the coming year, in order to allow the measurement at relevant low levels. In addition to the response of the liver equivalent, we also observed some reactivity in the human organotypic intestinal barrier. The mRNA expression of CYP $3 A 4$ decreased after treatment, in contrast to the increased levels in the liver tissue (Fig. 4D). The mRNA expression of NaK-ATPase decreased on (Fig 4 D). Similar to the data observed in the liver, MRP-1 and ZO-1 expression in the intestine barrier did not change during troglitazone treatment (Fig. 4D). Immunofluorescence staining could not observe any changes in NaK-ATPase, Cytokeratin $8 / 18$ or MRP2 expression, showing no effect on the polarisation of the tissue (Fig. 4A, B). To the best of our knowledge, there are no other comparable data available in the literature of such effects of troglitazone on human intestine, which makes the system an interesting research tool in this field. Finally, troglitazone treatment also reduced the expression of SGLT1 mRNA, but had no effect on PPAR-gamma, correlating to studies performed in rats[40].

Modeling systemic exposure to the liver-skin co-culture

A reproducible and easy to perform systemic substance administration protocol was performed over a stretch of nine days after a five-day administration-free adaptation period resulting in a continuous two-week MOC performance at repeated dose testing conditions. Systemic substance exposure to the MOC was modeled by administration of the respective troglitazone solution into the liver spheroid culture compartment of each MOC circuit. Here, we applied $50 \mu \mathrm{M}$ troglitazone, ten-fold the calculated systemic concentration in patients, to ensure the induction of metabolic effects within the liver equivalent both on mRNA and protein expression levels. Interestingly, the response was less pronounced than expected from earlier studies with $5 \mu \mathrm{M}$ troglitazone in non-endothelialized cocultures[19]. An increase in hepatocellular necrosis was detected in the cultures treated, demonstrated by TUNEL/Ki67 staining (Fig. 5A, B). Furthermore, an increase on the cytochrome P450 3A4 expression level was observed on the mRNA level (Fig. 5C), matching previous findings of an upregulated biotransformation activity following troglitazone treatment[19]. Troglitazone values in the media supernatants of the co-culture compared to respective single-tissue MOC cultures indicated that most of the substance was taken up by the tissues during the first days of treatment (Fig. 5D). It has been reported previously that troglitazone distributes extensively to various body tissues. The highest concentrations were reported to be in the liver, gastrointestinal tract and fat[30]. The uptake especially in the skin biopsies containing small amounts of fatty tissue underneath were observed in this study. Cultures containing liver equivalents also showed a reduction of troglitazone 
concentration in the media supernatants at the beginning of treatment, even though it was less prominent than in co-cultures containing skin biopsies. This might be due to the size difference between the respective tissue models. Skin biopsies had a mean volume of $24 \mu \mathrm{l}$, whereas liver equivalents had a volume of only 1 to $2 \mu \mathrm{l}$. The troglitazone concentration of liver single-tissue cultures and liver-endothelial co-cultures was similar (data not shown) and was always about $10 \mu \mathrm{M}$ below the concentration of endothelial single-tissue cultures in the MOC, indicating that that might be the concentration metabolized by the HepaRG cells every day. In the case of troglitazone, vascularisation was not necessary to avoid penetration into PDMS, as can be seen in Fig 5D: The total concentration of troglitazone in the medium in endothelial cell single-cultures, was $100 \%$ shortly after application. The culture of skin and liver showed a minimal decrease of troglitazone compared to the same culture with endothelial cells only in the first day of application and was equal in all following days. Thus, in our setting, a penetration of troglitazone into the PDMS with and without endothelial cells is minimal and can be ignored in this study.

\section{Conclusions}

Fourteen-day co-cultures of human 3D liver equivalents with human intestinal barriers, on the one hand, and skin biopsies, on the other hand, have successfully proven the capability of the microphysiological MOC system to reliably and reproducibly interconnect a parenchymal organ equivalent with various human barrier organ models. The MOC design assists a direct exposure of the liver equivalent to low shear stress, whilst shielding the human barrier organs from shear stress by industrial standard microporous insert membranes. The latter mode, in addition, facilitates the exposure of skin to the air-liquid interface. The co-cultures, operated at a total on-chip volume of 500 $\mu \mathrm{l}$ medium at recirculation rates of $2.6 \mu \mathrm{min}^{-1}$, stabilized at a metabolic steady state at day three for liver-intestine co-cultures and at day five for liver-skin co-cultures with an endothelialized microchannel system. Furthermore, the co-cultures sustained a repeated dose troglitazone exposure mimicking an oral administration route for the liver-intestine co-cultures and a systemic administration route for the endothelialized liver-skin co-culture. In both cases, the liver equivalents responded properly to the respective troglitazone challenge, as demonstrated at the mRNA and immunohistochemistry level. Interesting insights into histoarchitecture and metabolic reactivity of the barrier organs investigated could be gained regarding the homeostatic co-cultures. The stable maintenance of an entirely endothelialized microchannel system on the liver-skin MOCs and the successful establishment of the first in literature red blood cell assisted $\mu$ PIV measurement marked a milestone towards blood-perfused microphysiological MOCs. Finally, to the best of our knowledge, the troglitazone exposed liver-intestine co-cultures represent the first report of a long-term performance of a human primary columnar-like 3D epithelial barrier layer in microphysiological 
systems. The main advantage of this $\mathrm{MOC}$, is the possibility to culture two organ equivalents together in one system, where metabolites can be forwarded from one organ to another by a pulsatile flow. This enables interactions and crosstalk between the two organs. Barrier models can be inserted into the MOC for topical or apical application of substances to the system before reaching the second organ. Additionally, the effect of absorbance from one organ (e.g. the skin and its adipose tissue) can be taken into account. This may alter the available concentration of the substance in the medium, compared to the applied dose. Substance metabolites of one organ might have a different effect on the second organ than the initial substance applied, resulting in a different, but more precise outcome than in single cultures. Taken together, the MOC allows a more complex analysis of the effect on organs after substance exposure.

To summarize, our findings suggest that the MOC platform is capable of comprehensively maintaining various miniaturized human organ equivalents over long culture periods. Cells of different origin (i.e. primary cells, biopsies and cell lines) can be cultured, allowing a wide variety of applications. However, in order to standardise the system, iPS cells from the same donor, differentiated into different specific tissues, will be the most promising tissue source. This will also facilitate immune compatibility. MOC co-cultures can be exposed reproducibly to pharmaceutical substances at regimens and administration routes relevant to respective guidelines currently used for subsystemic substance testing in animals. In-depth analysis of the yet undefined fluid flow characteristics of the tissue culture compartments at current device geometries are ongoing to ground a fundamental understanding of the robust long-term stability of the liver and skin tissue cocultures reported here.

\section{Acknowledgements}

The work has been funded by the German Federal Ministry for Education and Research, GO-Bio Grant No. 0315569. We thank Dr. Guguen-Guillouzo, Dr. Gripon and Dr. Trepo who made HepaRG cells available. The small intestine tissue development was in part funded by NIH grant\# R43GM108164.

\section{References}

[1] U. Marx, H. Walles, S. Hoffmann, G. Lindner, R. Horland, F. Sonntag, et al., Human-on-a-chip Developments: A Translational Cutting-edge Alternative to Systemic Safety Assessment and Efficiency Evaluation of Substances in Laboratory Animals and Man, ATLA. 40 (2012) 235-57. http://www.ncbi.nlm.nih.gov/pubmed/23215661. 
[2] D. Huh, Y. Torisawa, G. a Hamilton, H.J. Kim, D.E. Ingber, Microengineered physiological biomimicry: organs-on-chips., Lab Chip. 12 (2012) 2156-64. doi:10.1039/c2lc40089h.

[3] M.B. Esch, A.S.T. Smith, J. Prot, C. Oleaga, J.J. Hickman, M.L. Shuler, How multi-organ microdevices can help foster drug development is, Adv. Drug Deliv. Rev. 69-70 (2014) 158169. doi:10.1016/j.addr.2013.12.003.

[4] K.M. Fabre, C. Livingston, D. a Tagle, Organs-on-chips (microphysiological systems): tools to expedite efficacy and toxicity testing in human tissue., Exp. Biol. Med. (Maywood). 239 (2014) 1073-7. doi:10.1177/1535370214538916.

[5] J.P. Wikswo, The relevance and potential roles of microphysiological systems in biology and medicine., Exp. Biol. Med. (Maywood). 239 (2014) 1061-72. doi:10.1177/1535370214542068.

[6] E.-M. Materne, A.G. Tonevitsky, U. Marx, Chip-based liver equivalents for toxicity testing-organotypicalness versus cost-efficient high throughput., Lab Chip. 13 (2013) 3481-95. doi:10.1039/c3lc50240f.

[7] J. Hwan, M.L. Shuler, J.H. Sung, A micro cell culture analog (microCCA) with 3-D hydrogel culture of multiple cell lines to assess metabolism-dependent cytotoxicity of anti-cancer drugs., Lab Chip. 9 (2009) 1385-94. doi:10.1039/b901377f.

[8] J.H. Sung, C. Kam, M.L. Shuler, A microfluidic device for a pharmacokinetic-pharmacodynamic (PK-PD) model on a chip., Lab Chip. 10 (2010) 446-55. doi:10.1039/b917763a.

[9] C. Zhang, Z. Zhao, A. Abdul, V. Noort, H. Yu, N.A. Abdul Rahim, et al., Towards a human-onchip: culturing multiple cell types on a chip with compartmentalized microenvironments., Lab Chip. 9 (2009) 3185-92. doi:10.1039/b915147h.

[10] Y. Imura, K. Sato, E. Yoshimura, Micro total bioassay system for ingested substances: assessment of intestinal absorption, hepatic metabolism, and bioactivity., Anal. Chem. 82 (2010) 9983-8. doi:10.1021/ac100806x.

[11] T. Bricks, P. Paullier, A. Legendre, M.-J. Fleury, P. Zeller, F. Merlier, et al., Development of a new microfluidic platform integrating co-cultures of intestinal and liver cell lines., Toxicol. In Vitro. 28 (2014) 885-95. doi:10.1016/j.tiv.2014.02.005.

[12] H. Mertsching, J. Schanz, V. Steger, M. Schandar, M. Schenk, J. Hansmann, et al., Generation and transplantation of an autologous vascularized bioartificial human tissue., Transplantation. 88 (2009) 203-10. doi:10.1097/TP.0b013e3181ac15e1.

[13] J. Schanz, J. Pusch, J. Hansmann, H. Walles, Vascularised human tissue models: A new approach for the refinement of biomedical research, J. Biotechnol. 148 (2010) 56-63. doi:10.1016/j.jbiotec.2010.03.015.

[14] K. Scheller, I. Dally, N. Hartman, B. Münst, J. Braspenning, H. Walles, Upcyte Microvascular Endothelial Cells Repopulate Decellularized Scaffold, Tissue Eng. Part C Methods. 19 (2013) 57-67.

[15] J.H. Yeon, H.R. Ryu, M. Chung, Q.P. Hu, N.L. Jeon, In vitro formation and characterization of a perfusable three-dimensional tubular capillary network in microfluidic devices., Lab Chip. 12 (2012) 2815-22. doi:10.1039/c2lc40131b. 
[16] S. Kim, H. Lee, M. Chung, N.L. Jeon, Engineering of functional, perfusable 3D microvascular networks on a chip., Lab Chip. 13 (2013) 1489-500. doi:10.1039/c3lc41320a.

[17] H. Lee, S. Kim, M. Chung, J.H. Kim, N.L. Jeon, A bioengineered array of 3D microvessels for vascular permeability assay., Microvasc. Res. 91 (2014) 90-8. doi:10.1016/j.mvr.2013.12.001.

[18] K. Schimek, M. Busek, S. Brincker, B. Groth, S. Hoffmann, R. Lauster, et al., Integrating biological vasculature into a multi-organ-chip microsystem., Lab Chip. 13 (2013) 3588-98. doi:10.1039/c3lc50217a.

[19] I. Wagner, E.-M. Materne, U. Marx, S. Brincker, U. Süssbier, C. Frädrich, et al., A dynamic multi-organ-chip for long-term cultivation and substance testing proven by $3 D$ human liver and skin tissue co-culture., Lab Chip. 13 (2013) 3538-47. doi:10.1039/c3lc50234a.

[20] M.-H. Wu, S. Huang, Z.Z. Cui, G.-B. Lee, Development of perfusion-based micro 3-D cell culture platform and its application for high throughput drug testing, Sensors Actuators B Chem. 129 (2008) 231-240. doi:10.1016/j.snb.2007.07.145.

[21] M.J. Powers, K. Domansky, M.R. Kaazempur-Mofrad, A. Kalezi, A. Capitano, A. Upadhyaya, et al., A Microfabricated Array Bioreactor for Perfused 3D Liver Culture, Biotechnol. Bioeng. 78 (2002) 257-269. doi:10.1002/bit.10143.

[22] W. Thielicke, E.J. Stamhuis, PIVlab - Towards User-friendly, Affordable and Accurate Digital Particle Image Velocimetry in MATLAB, J. Open Res. Softw. 2 (2014) e30. doi:10.5334/jors.bl.

[23] W. Thielicke, The Flapping Flight of Birds - Analysis and Application, 2014.

[24] V. Gupta, N. Doshi, S. Mitragotri, Permeation of insulin, calcitonin and exenatide across Caco2 monolayers: measurement using a rapid, 3-day system., PLoS One. 8 (2013) e57136. doi:10.1371/journal.pone.0057136.

[25] P. Artursson, A.-L. Ungell, J.-E. Löfroth, Selective paracellular permeability in two models of intestinal absorption: cultured monolayers of human intestinal epithelial cells and rat intestinal segments, Pharm. Res. 10 (1993) 1123-1129.

[26] E. Le Ferrec, C. Chesne, P. Artusson, D. Brayden, G. Fabre, P. Gires, et al., In Vitro Models of the Intestinal Barrier, ATLA. 29 (2001) 649-668.

[27] P. Gripon, S. Rumin, S. Urban, J. Le Seyec, D. Glaise, I. Cannie, et al., Infection of a human hepatoma cell line by hepatitis B virus., Proc. Natl. Acad. Sci. U. S. A. 99 (2002) 15655-60. doi:10.1073/pnas.232137699.

[28] J. Yu, S. Peng, D. Luo, J.C. March, In vitro 3D human small intestinal villous model for drug permeability determination., Biotechnol. Bioeng. 109 (2012) 2173-8. doi:10.1002/bit.24518.

[29] J.H. Sung, J. Yu, D. Luo, M.L. Shuler, J.C. March, Microscale 3-D hydrogel scaffold for biomimetic gastrointestinal (GI) tract model., Lab Chip. 11 (2011) 389-92. doi:10.1039/c0lc00273a.

[30] C.M. Loi, M. Young, E. Randinitis, A. Vassos, J.R. Koup, Clinical pharmacokinetics of troglitazone, Clin. Pharmacokinet. 37 (1999) 91-104. doi:10.2165/00003088-19993702000001. 
[31] A. Rogue, C. Lambert, R. Jossé, S. Antherieu, C. Spire, N. Claude, et al., Comparative gene expression profiles induced by PPAR $\gamma$ and PPAR $/ \gamma$ agonists in human hepatocytes., PLoS One. 6 (2011) e18816. doi:10.1371/journal.pone.0018816.

[32] A. Limonciel, L. Aschauer, A. Wilmes, S. Prajczer, M.O. Leonard, W. Pfaller, et al., Lactate is an ideal non-invasive marker for evaluating temporal alterations in cell stress and toxicity in repeat dose testing regimes, Toxicol. In Vitro. 25 (2011) 1855-62. doi:10.1016/j.tiv.2011.05.018.

[33] H. Yamazaki, A. Shibata, M. Suzuki, M. Nakajima, N. Shimada, F.P. Guengerich, et al., OXIDATION OF TROGLITAZONE TO A QUINONE-TYPE METABOLITE CATALYZED BY CYTOCHROME P-450 2C8 AND P-450 3A4 IN HUMAN LIVER MICROSOMES ABSTRACT:, 27 (1999) 1260-1266.

[34] T. Izumi, K. Hosiyama, S. Enomoto, K. Sasahara, Y. Sugiyama, Pharmacokinetics of Troglitazone, an Antidiabetic Agent: Prediction of In Vivo Stereoselective Sulfation and Glucuronidation from In Vitro Data, 280 (1997) 1392-1400.

[35] H.E. Cubitt, J.B. Houston, A. Galetin, Prediction of Human Drug Clearance by Multiple Metabolic Pathways: Integration of Hepatic and Intestinal Microsomal and Cytosolic Data, (2011) 864-873. doi:10.1124/dmd.110.036566.

[36] T.I. Yasushi YOSHIGAE, Kumiko KONNO, Wataru TAKASAKI, CHARACTERIZATION OF UDPGLUCURONOSYLTRANSFERASES(UGTS)INVOLVED IN THE METABOLISM OF TROGLITAZONE IN RATS AND HUMANS, J. Toxicol. Sci. 25 (2000) 433-441. http://dx.doi.org/10.2131/jts.25.5_433.

[37] C. Funk, C. Ponelle, G. Scheuermann, M. Pantze, Cholestatic Potential of Troglitazone as a Possible Factor Contributing to Troglitazone-Induced Hepatotoxicity: In Vivo and in Vitro Interaction at the Canalicular Bile Salt Export Pump (Bsep) in the Rat, Mol. Pharmacol. 59 (2001) 627-635.

[38] R. Kaimal, X. Song, B. Yan, R. King, R. Deng, Differential Modulation of Farnesoid X Receptor Signaling Pathway by the Thiazolidinediones, J. Pharmacol. Exp. Ther. 330 (2009) 125-134. doi:10.1124/jpet.109.151233.PPAR.

[39] Rogue et al. - 2011 - Comparative gene expression profiles induced by PPARY and PPARay agonists in human hepatocytes.pdf, (n.d.).

[40] C. Corpe, S. Sreenan, C. Burant, Effects of Type-2 Diabetes and Troglitazone on the Expression Patterns of Small Intestinal Sugar Transporters and PPAR-Gamma in the Zucker Diabetic Fatty Rat, Digestion. 63 (2001) 116-123. 


\begin{tabular}{|c|c|c|c|}
\hline \multicolumn{2}{|l|}{ Gene } & \multirow{2}{*}{$\begin{array}{l}\text { Primer sequences }\left(5^{\prime}-\mathbf{3}^{\prime}\right) \\
\text { TGCAAGGCTGACGATAAGGAG }\end{array}$} & \multirow{2}{*}{$\begin{array}{l}\text { Primers used } \\
\text { for RT-qPCR }\end{array}$} \\
\hline \multirow[t]{2}{*}{ Albumin } & $\mathrm{F}$ & & \\
\hline & $\mathrm{R}$ & TTTAGACAGGGTGTTGGCTTTACAC & \\
\hline \multirow[t]{2}{*}{ Cyp $3 A 4$} & $\mathrm{~F}$ & GGAAGTGGACCCAGAAACTGC & \\
\hline & $\mathrm{R}$ & TTACGGTGCCATCCCTTGAC & \\
\hline \multirow[t]{2}{*}{ BSEP } & $\mathrm{F}$ & GCAGACACTGGCGTTTGTTG & \\
\hline & $\mathrm{R}$ & ATGTTTTAAGGGAGGAACTGG & \\
\hline \multirow[t]{2}{*}{ MRP-1 } & $F$ & ACCTCATCCAGTCCACCATCC & \\
\hline & $\mathrm{R}$ & GGCCATGCTGTAGAAAAGACCTC & \\
\hline \multirow[t]{2}{*}{$20-1$} & $\mathrm{~F}$ & TCTCGGAAAAGTGCCAGGAAG & \\
\hline & $\mathrm{R}$ & CCCTCGGAAACCCATACCAG & \\
\hline \multirow[t]{2}{*}{ CPS-1 } & $\mathrm{F}$ & CCCAGCCTCTCTTCCATCAG & \\
\hline & $\mathrm{R}$ & GCGAGATTTCTGCACAGCTTC & \\
\hline \multirow[t]{2}{*}{ PPARa } & $\mathrm{F}$ & ACAAGGTCAAAGCCCGGGTC & \\
\hline & $\mathrm{R}$ & AGCAGTGAAAGATGCGGACCT & \\
\hline \multirow[t]{2}{*}{ MMP7 } & $\mathrm{F}$ & TGGGGAACTGCTGACATCATGA & \\
\hline & $\mathrm{R}$ & TCCCTAGACTGCTACCATCCGT & \\
\hline \multirow[t]{2}{*}{ PPARg } & $\mathrm{F}$ & CCCAGGTTTGCTGAATGTGAAG & \\
\hline & $\mathrm{R}$ & GAAGGGAAATGTTGGCAGTGG & \\
\hline \multirow[t]{2}{*}{ MUC 2} & $\mathrm{~F}$ & CCGACCTCCAGCACAGTTTT & \\
\hline & $\mathrm{R}$ & GATCCTTGACCGACCTGCAC & \\
\hline \multirow[t]{2}{*}{ NaK-ATPase } & $\mathrm{F}$ & ACAGCCCAGAAATCCCAAAAC & \\
\hline & $\mathrm{R}$ & CAGCGGTCATCCCAGTCC & \\
\hline \multirow[t]{2}{*}{ SLC5A1 } & $\mathrm{F}$ & GCATCTCTACCGTCTGTGTTGG & \\
\hline & $\mathrm{R}$ & TGCTGCTCTAGCCCACAAAAT & \\
\hline \multirow[t]{2}{*}{ Villin } & $\mathrm{F}$ & GGCCTCTGCCCATCTTCC & \\
\hline & $\mathrm{R}$ & AGGTTTTGTTGCTTCCATCGAG & \\
\hline \multirow[t]{2}{*}{ TBP } & $\mathrm{F}$ & CCTTGTGCTCACCCACCAAC & \\
\hline & $\mathrm{R}$ & TCGTCTTCCTGAATCCCTTTAGAATAG & \\
\hline
\end{tabular}


Figure 1. Tissue allocation and microfluidic profiling in the MOC. A, B) Cross section of an assembled MOC schematically illustrating the liver-intestine co-culture $(A)$ and liver-skin co-culture (B) in the right-hand circuit of each MOC. Emulation of systemic substance exposure is illustrated in the liver compartment of A, whilst mimicry of oral administration is shown on the intestine (B). C) Clockwise and counterclockwise fluid flow within the two circuits is illustrated by bold arrows. Red blood cell assisted $\mu$ PIV measurements in position A of the microchannels at different process conditions support selection of optimal flow conditions. D) Example of a pulsatile flow profile of human red blood cells in position A of an endothelialized MOC. Median flow rates and median shear stress values were calculated on the basis of this profile. 
Figure 2. Performance of liver-intestine co-cultures in MOCs over 14 days. Staining for A) TUNEL/Ki67 showing apoptosis (green) and viability (red) in small intestinal epithelial tissue. B) MRP2 (red) expression in small intestinal epithelial tissues. C) Transporter NaK-ATPase (red) and epithelial cells cytokeratin 8/18 (green) in small intestinal epithelial tissues. D) Equal distribution of HHSteC, stained with vimentin (red), within hepatocytes, stained with cytokeratin 8/18(green). (A-D) Nuclei were stained with DAPI (blue). 
Figure 3. Performance of liver-skin co-cultures in endothelialized MOCs over 14 days. A) Calcein AM staining (red) showed viable and evenly distributed HDMECs in all areas of the microfluidic circuit. B) Higher magnification view of HDMEC monolayer. C) HDMEC-liver tissue interaction shown by cytokeratin 8/18 (red) staining of hepatocytes and VWF (green) staining of HDMECs. D) Skin biopsy stained for cytokeratin 15 (red) and cytokeratin 10 (green) showing a preserved continuous layer of cells. Nuclei were stained with Hoechst (blue). Scale bars $100 \mu \mathrm{m}$. E) Glucose and lactate metabolic activity of co-cultures of skin, liver and endothelial cells over 14 days. 
Figure 4. Performance of liver and small intestinal epithelial tissue co-cultures in MOCs after 11 days of chronic troglitazone exposure. A) Staining of small intestinal epithelial tissue for transporter NaK-ATPase (red) and cytokeratin 8/18 (green). B) Staining of small intestinal epithelial tissue for the ATP-dependent export pump MRP-2 (red). C) qRT-PCR data of liver tissues from control (normalized to 1) and treated co-cultures analysed for expression of Albumin, BSEP, CPS1, Cyp3A4, MMP7, MRP1, PPARa, UGT1A1 and ZO-1. D) qRT-PCR data of small intestinal tissues from control (normalized to 1) and treated co-cultures analysed for expression of CYP3A4, PPARg, MRP-1, MUC2, NaK-ATPase, SLC5A1, Villin, and ZO-1. (A and B) Nuclei were stained with DAPI (blue). Scale bars $100 \mu \mathrm{m}$. (C and D) Data are mean \pm SD of four independent co-cultures. * indicates $p \leq 0.05$ 
Figure 5. Performance of liver-skin co-cultures in endothelialized MOCs after exposure to troglitazone. TUNEL/Ki67 staining of liver equivalents from co-cultures that were A) treated as control or B) exposed to $50 \mu \mathrm{M}$ troglitazone. Scale bars $300 \mu \mathrm{m}$. C) qRT-PCR data of liver tissues from control and treated co-cultures analyzed for expression of Albumin, Cyp3A4, BSEP, MRP-1, ZO-1 and CPS-1. Data are mean \pm SD of four independent co-cultures. * indicates $p \leq 0.05$ D) Troglitazone concentration in the media supernatants of respective single tissue and co-cultures over time. MOC Li: liver single tissue culture in the MOC; MOC Sk: skin single tissue culture in the MOC; MOC Va: endothelial single-tissue culture in the MOC; and MOC Li-Va-Sk: respective three-tissue co-culture. 


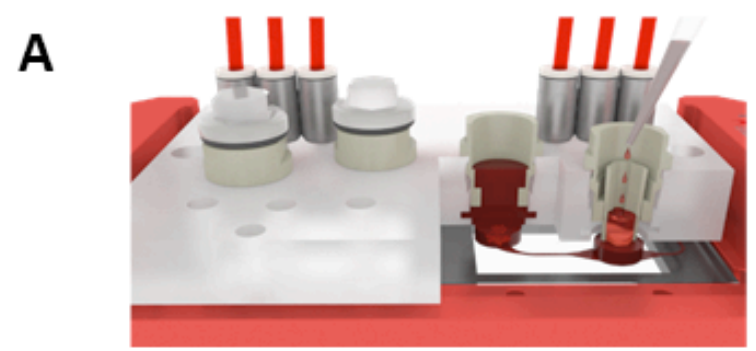

C on-chip micropump

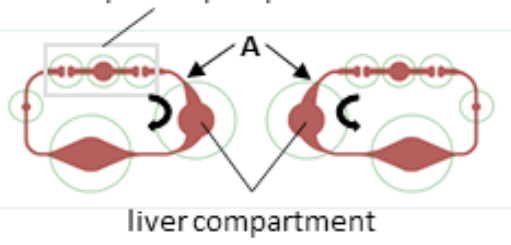

B

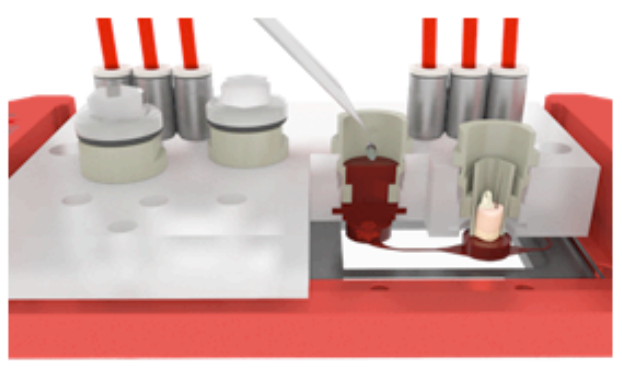

D

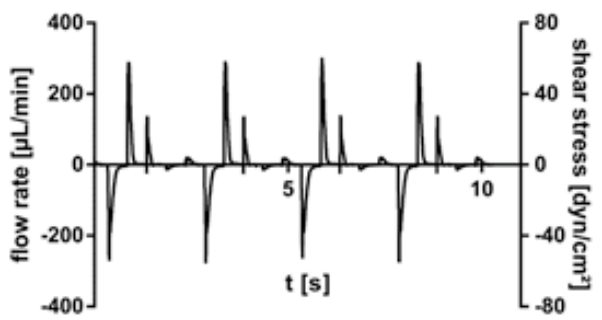

Figure 1 

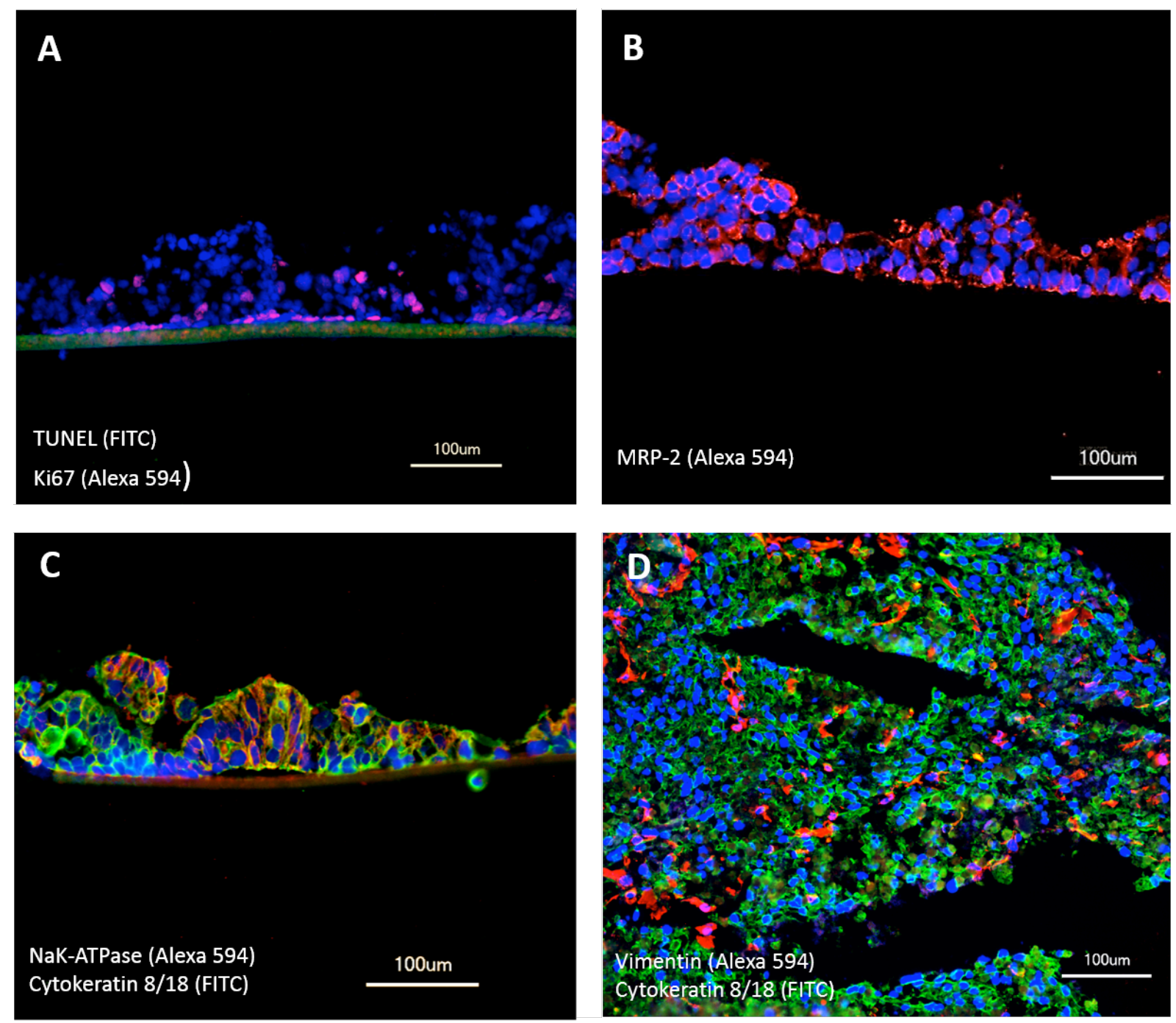

Figure 2 


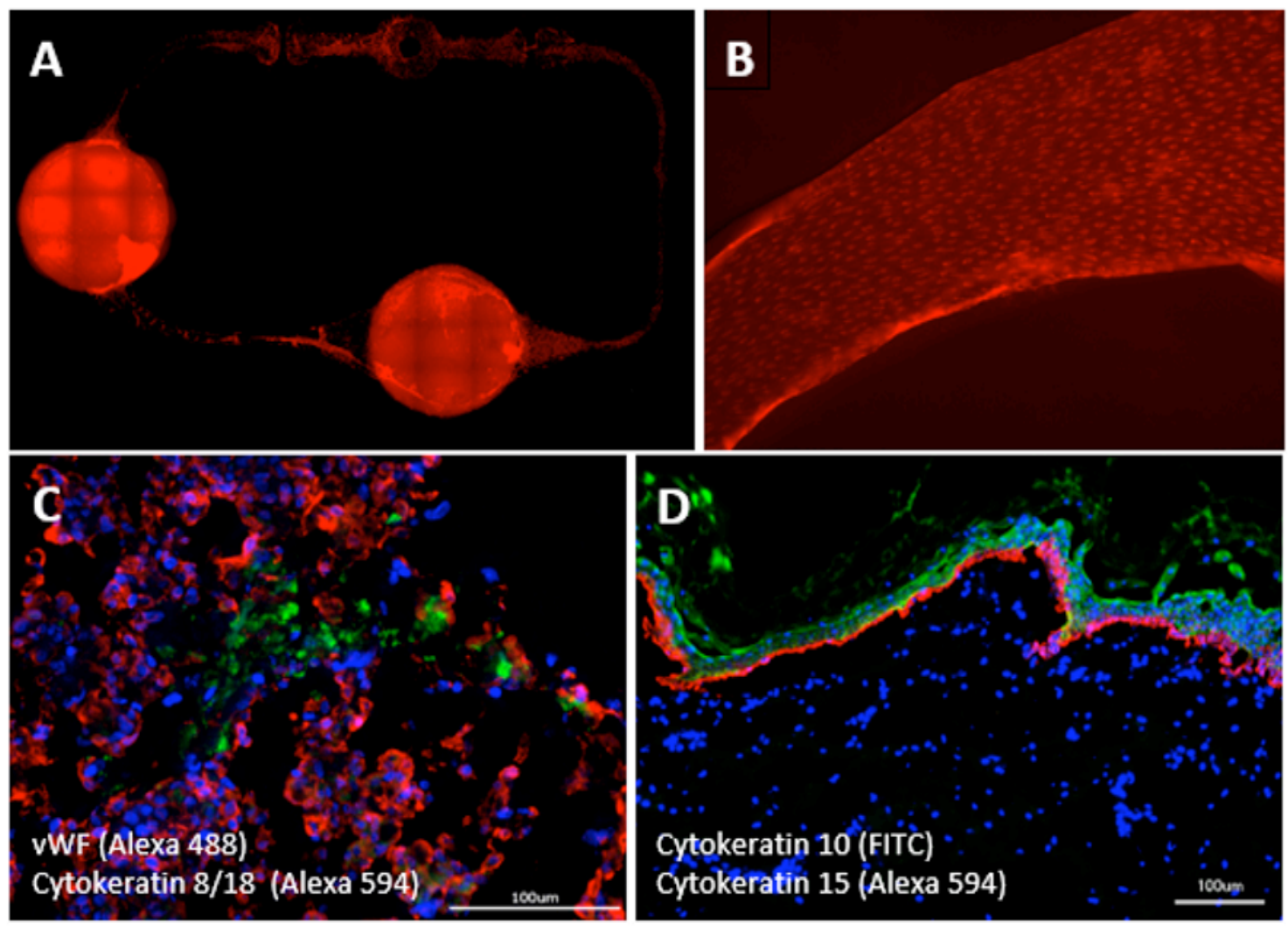

E

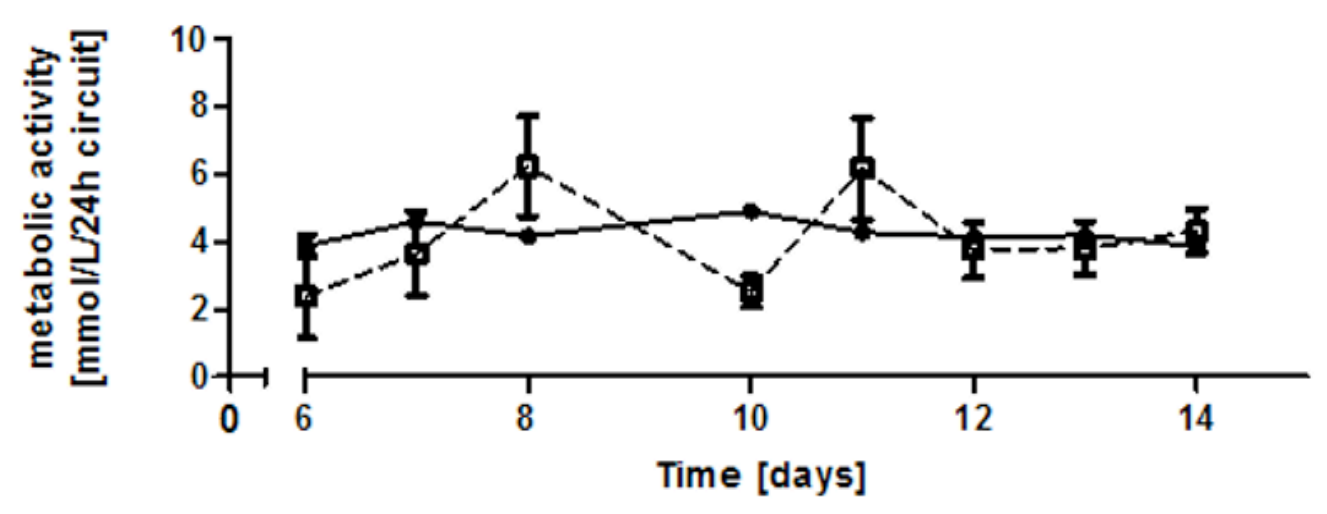

-c. Lactate $\rightarrow$ Glucose

Figure 3 

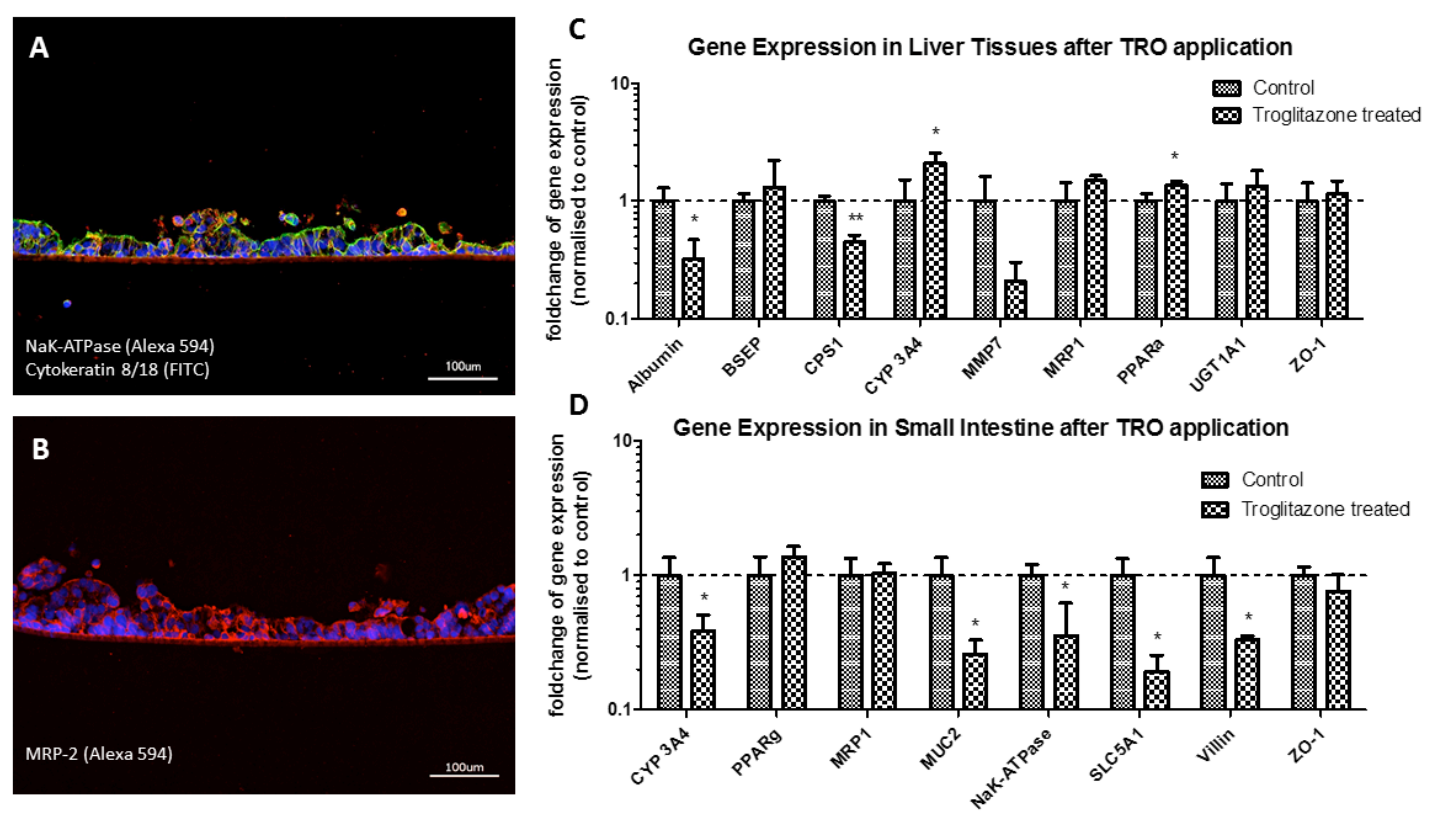

Figure 4 

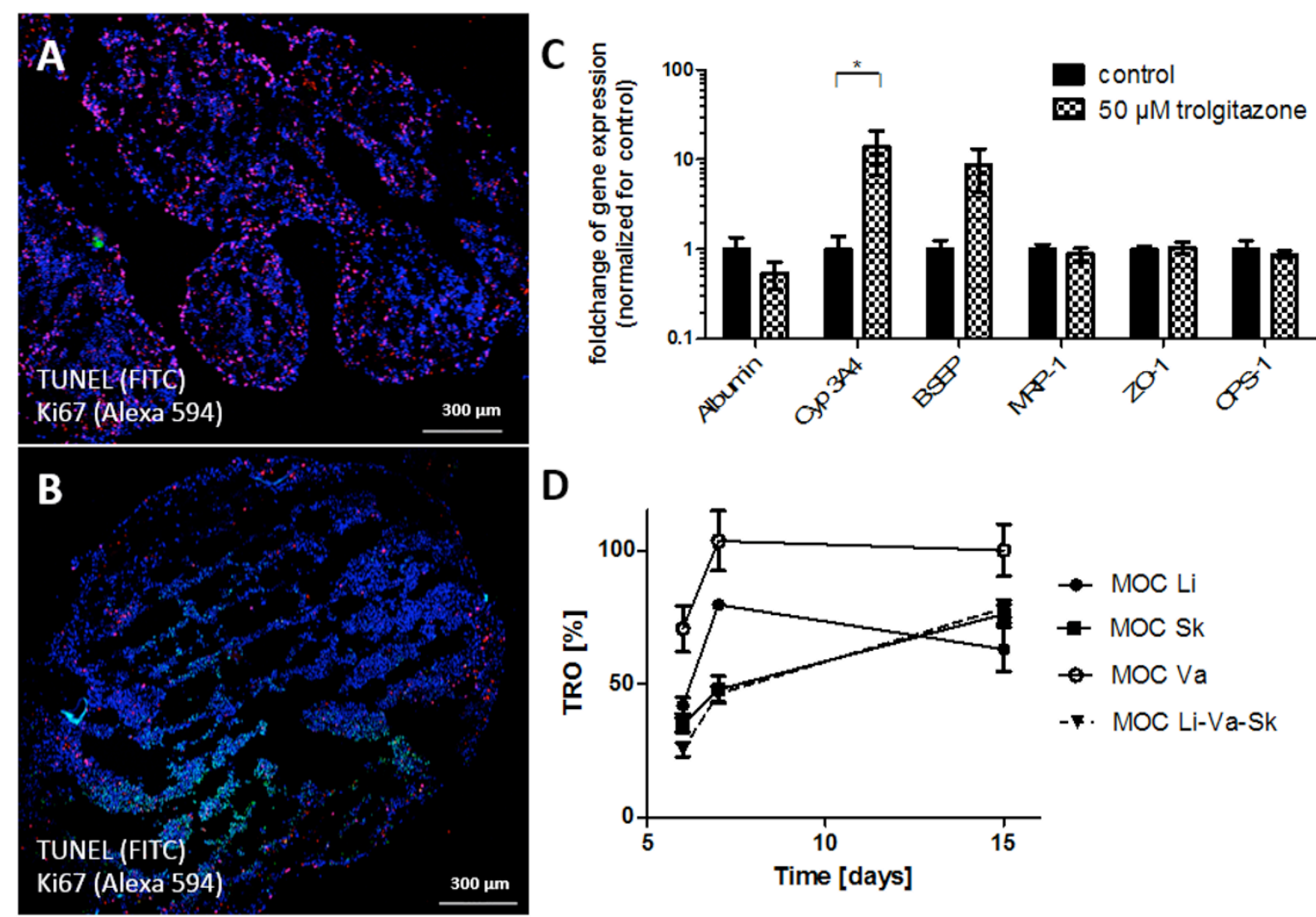

Figure 5 